\title{
System Dynamics-Based Analysis of Dynamic Mechanism on E-commerce Industry
}

\author{
Haiying $\mathrm{Ma}^{*}$ \\ School of Economics, Northwest Minzu University \& Provincial Key Laboratory of E-commerce of \\ Ethnic Information, Northwest Minzu University, Lanzhou (730124), P.R.China \\ Ixmahaiying8888@163.com
}

\section{Keywords: E-commerce industry; System Dynamics; Complex adaptive system}

\begin{abstract}
E-commerce is a new type of economic activity that is highly commensurate with modern information technology and business activities. It has become an important force that leads the transformation of production and lifestyle. The rapid development of E-commerce in recent years has become an important component of China's strategic emerging industries. It is an important tool for achieving the Internet Plus strategy, and it is advancing the transformation of economic growth methods. Structural adjustments and other aspects have played a key role and has increasingly become an important engine for promoting China's economic transformation and upgrading. At the same time, the development of E-commerce in China still faces contradictions and problems such as non-standard market systems, imperfect legal systems, and imperfect logistics support. This requires in-depth analysis of the development of E-commerce. Firstly, this paper applies the theory of complex adaptive systems to analyze the dynamic mechanism of E-commerce industry development. Then, it uses the system dynamics method to construct the dynamic learning model of E-commerce industry development system and analyzes the trend characteristics of E-commerce development. The model consists of technological innovation and market. There are four interrelated subsystems: demand, economic development, and industrial environment.
\end{abstract}

\section{Introduction}

In recent years, the rapid development of E-commerce in China has developed into an important component of the country's strategic emerging industries. It has extensively penetrated into the fields of production, circulation, and consumption. The depth of integration with traditional industries has been relentless, and social needs have become increasingly embryonic. The important field of the Internet Plus strategy has played an active role in promoting economic restructuring and structural adjustment, and is becoming an important engine for promoting China's economic transformation and upgrading. During the 11th Five-Year Plan period, the country vigorously supported the development of E-commerce and issued the relevant development and planning policy documents for the first time, including the Eleventh Five-Year Development Plan for E-commerce, which made a clear deployment to the development of China's E-commerce industry and provided policy guarantees. E-commerce industry in China has been advancing rapidly during the 11th Five-Year Plan period and has gradually become a new bright spot in economic development. During the 12th Five-Year Plan period, it was identified as a strategic emerging industry and faced with good opportunities for development. Statistics show that in 2015, China's E-commerce transactions amounted to 20.8 trillion Yuan, 27\% year-on-year, accounting for $30 \%$ of GDP, and made great contributions to the development of the national economy. In 2015, the State Council issued the Opinions on Developing E-commerce and Accelerating the Cultivation of New Economic Action, pointing out that E-commerce has become the new driving force for economic development, but E-commerce itself is faced with unmanageable management methods and irregular market order. Problems such as institutional mechanisms need to grasp the new trends and directions of E-commerce development and vigorously promote policy innovation, management innovation, and service innovation. As the Internet Plus strategy are put forward and in-depth implementation, as an important link, the development of the E-commerce industry will be of great 
interest. The year 2016 is the first year of the Eleventh Five-Year Plan period. It is necessary to actively play the role of E-commerce in promoting the upgrading of traditional industries and achieve a positive interaction with economic development.

Therefore, in order to realize the healthy development of E-commerce, we need to deeply analyze the development status and development mechanism of E-commerce in China, explore the intrinsic relationship between E-commerce and economic development, study the development trend of E-commerce industry, and forecast the E-commerce industry in different industrial policy environments. This has important practical significance for optimizing the development environment of China's E-commerce and further enhancing the quality and efficiency of economic development. Based on the research of E-commerce industry development, this article reviews the E-commerce industry, applies the complex adaptation system and system dynamics theory and methods, analyzes the dynamic mechanism of E-commerce industry development, and then builds the E-commerce industry development system. The dynamic model analyzes the interaction between the E-commerce industry and economic development.

\section{System Dynamics Applied in Electronic Commerce}

System dynamics is widely used to study economic management issues, and it is also effective in solving problems in E-commerce. Yu and Yang (2008) used the analysis theory of system dynamics to construct a model of agricultural E-commerce development dynamic system and explored the influencing factors of agricultural E-commerce development. Zhang (2010) used system dynamics to model the operation of the mobile E-commerce industry chain, and analyzed in depth the constraints of the mobile E-commerce payment problem. Zhang (2013) used system dynamics methods to model and analyze E-commerce third-party logistics, and simulated the historical data of Taobao. Xu (2013) applied the theory of system dynamics to study the dynamic mechanism of agricultural E-commerce development. Based on this, it constructed an evaluation index for the development of E-commerce in agriculture and provided theoretical support for studying the development level of E-commerce. Ma (2014) uses system dynamics to establish a dynamic model of word of mouth and purchase intention in online shopping, and analyzes the importance of E-commerce consumer experience and word of mouth. E-commerce is a dynamic development system, in which the elements change with time and space. The application of system dynamics to the research of industrial development system proves its unique superiority. It is feasible to study the development of E-commerce industry by using system dynamics.

\section{The Analysis of the Dynamic Mechanism on E-commerce Industry Development}

Dynamics Mechanism refers to the motive force and mode of action that promote the development of things. It is a mechanism that can promote the system to achieve sustainable development and achieve the intended goal to provide incentives. From a systems theory perspective, the E-commerce industry is an open and complex system that continues to evolve over time as the internal driving forces interact with the external environment. The followings analyze the dynamic mechanism of E-commerce industry development from the perspective of complex adaptive system theory.

The Basic Characteristics of CAS in E-commerce Industry. The development of the E-commerce industry not only depends on the interaction within the industry, but also on the relationship between the external social and economic environment and the ability of the system to adapt to the economic environment. Therefore, the E-commerce industry needs to reluctantly adapt itself to the economy and achieve its own development while promoting the transformation and development of the national economy. The current E-commerce development management model faces complex challenges, and the complex adaptive system theory has important guiding significance for solving complex system problems and phenomena. Studying industrial development as a complex system has become a new research trend in modern management science. Using its own research results to explore the dynamic mechanism of E-commerce industry development is a useful attempt. 
Aggregation Features. The smaller entities in a complex adaptive system are better developed through aggregation. The main bodies of the E-commerce industry system include the government, E-commerce companies, and E-commerce service providers. The various E-commerce entities will gather for specific goals. For example, in order to integrate China's logistics resources and achieve the sharing of resources, Alibaba took advantage of the scale effect of E-commerce logistics companies, gathered logistics companies.

Non-linear Features. Non-linearity refers to the change of the internal body of the system and the interaction mechanism between the subject and the environment does not follow the linear principle. The internal relationship of the E-commerce industry system is complex and complex, and when the subject changes, it will have dynamic and complex non-linear relationships. E-commerce is a product of the network information economy, and the network economy is essentially non-linear. In the E-commerce industry chain, the government, enterprises, service providers, consumers and other entities all have their own goals and behaviors, not in the interaction process. Simple causal relationships form a variety of forward and reverse complex feedback chains. At the same time, there are many random factors in the E-commerce industry system, which makes the E-commerce industry development process unpredictable and irreversible. This unpredictability and uncertainty trigger the system's nonlinearity. In short, the main body in the E-commerce industry system is not a simple linear relationship, but a multi-level, multi-objective, multi-result nonlinear relationship.

Flow Characteristics. Flows in a complex adaptive system refer to the flow of resources between the entities. The smoothness of the flow channels relates to the efficiency of system development. In the E-commerce industry system, there is a large amount of information flow, capital flow, and logistics. For example, in the online shopping process, consumers understand the information of the required goods through the E-commerce platform, and then through the payment of funds, after the enterprise receives the order information, the goods are delivered to the consumers through logistics. It is the activities of various streams that inject constant energy to drive the good running of the E-commerce industry. The flow of substances, capabilities, and information in the system shows that the E-commerce industry system has a flow characteristic.

Diversity Features. The diversified features of the E-commerce industry system are very prominent. It is a new product resulting from the interaction of the E-commerce industry system with politics, economy, and culture. For example, due to changes in the internal and external environment, the E-commerce business model has various modes such as B2B, B2C, C2C, and C2B.

Identification Mechanism. The identification can promote the purposeful choice of interactive objects of the system subject, and promote the combination of subjects with a synergistic effect. The aggregation of the subject is performed under the function of the "identification" mechanism. In the process of forming the E-commerce industry chain, its own goals, resources, and advantages are gathered for the logos. The various stakeholders choose to collaborate with their own development partners to form complementary advantages and create a strong industrial chain to respond to external competitors or Market risk.

Internal Model Mechanism. The internal model is a behavioral rule that the subject gradually establishes in the process of adapting to the environment. The adaptive behavior is selective, selective reception of external stimuli, and optimization of internal mechanisms to barely respond to environmental changes. In the development process of the E-commerce industry, it will constantly go in with other industries, continue to grow in the process of adapting to the environment, and form its own internal response mechanism, thus becoming a new industry.

Building Block Mechanism. Building blocks are the elements that make up a complex system. The different combinations and quantities of building blocks determine the complexity of the system. The E-commerce industry system consists of companies, governments, service organizations, scientific research institutions, and other building blocks. When they coexist for specific purposes, they can form industrial chains, industrial parks, and other forms. These blocks interact and remain independent, serving as different functions for the development of the system. As a profitable economic organization, an enterprise is the main promoter of the development of the E-commerce industry; the government 
provides policy and financial support to intervene in the E-commerce industry system in a way that regulates and guides; universities and scientific research institutions mainly carry out scientific and technological innovation research, and deliver cutting-edge technologies to enterprises. Technology; service organizations mainly include financial and logistics institutions. Financial institutions solve the financing problems of enterprises in the course of E-commerce activities, and logistics companies solve the problem of transportation of goods. According to the industrial development strategy, the introduction of relevant E-commerce companies and service organizations to form industrial parks is a combination of building blocks.

Dynamic Mechanism of E-commerce Industry Development. The stimulus-response model is a basic model that is often used to analyze the dynamics of system development in the theory of complex adaptive systems. It describes the evolution of the system. The model consists of three parts: the detector set, the rule set, and the effectors set. The basic principle is that the subject senses external environmental stimuli through the detector, passes the classification filter and passes it to the internal model, and the internal model matches with the rule set. Out of coping strategies, directly activate the effectors to generate behavior. The E-commerce industry involves a wide range, strong penetration, and the external motivation of the detector is complex, mainly including market demand, government support, and industrial environment. After receiving the external stimuli, the system's internal subjects are identified and processed and passed to the internal model. The intrinsic dynamics of the main body include the promotion of technological innovation and the driving force of economic benefits.

Intrinsic Power Mechanism. (1) Economic benefits. With the advancement of E-commerce technology, E-commerce has penetrated into all walks of life in the mad society, and has brought about a positive role in promoting the economic efficiency of enterprises and the longevity of the national economy. The economic benefits of E-commerce are represented by microscopic corporate profits and macroscopic economic growth. At the micro level, as a profitable economic organization, enterprises use E-commerce to conduct business activities, which can reduce business costs and improve the operating efficiency of enterprises, thus ensuring survival and development of the enterprise, the E-commerce company's pursuit of interests is the internal driving force to promote the development of the E-commerce industry. (2) Technology Innovation. The progress of science and technology from within the E-commerce industry to provide them with development opportunities and support for the transformation of business models is an internal driving factor in the development of E-commerce. The adoption of advanced technologies can promote the development of E-commerce from two aspects: First, the combination of the Internet and traditional businesses has spawned E-commerce. With the continuous improvement of the level of science and technology, the development of cloud computing and big data technologies has promoted the E-commerce operating model.

External Power Mechanism. (1) Market demand. With the development of the economy, market demand will show diversity and dynamic changes. The development of the E-commerce industry will require strong market demand. The formation of the E-commerce industry is due to the fact that it meets the consumer demands of modern consumers and meets the characteristics of the social environment. The demand for the E-commerce market is huge, including the sum of the E-commerce needs of individuals, businesses, governments, and other organizations. (2) Government support. E-commerce is a new thing. At the same time, it is also a strategic emerging industry of the country. Its technological development speed and business model have changed rapidly, and its role in promoting the transformation and development of the economy has become obvious. It has become a new driving force for economic growth. Government policy Promoting and guiding is an important and indispensable quantity for the development of the E-commerce industry.

\section{Conclusions and Suggestions}

The development of the E-commerce industry is a complex and dynamic process, but mastering the key factors that affect the development of E-commerce, clarifying the interaction mechanism between E-commerce and economic growth, and simplifying the complexity of E-commerce industry development analysis. Based on the existing researches, this paper uses the complex adaptive system 
theory to analyze the dynamic mechanism of the development of the E-commerce industry, and further builds a dynamic model for the development of the E-commerce industry. It analyzes the causality of various variables within the E-commerce industry development system and divides the system into points. For the market demand subsystem, technological progress subsystem, economic development subsystem, and industrial environment subsystem, the interactive relationship between E-commerce and economic development is studied. Based on the data of China's E-commerce industry, the trend characteristics of China's E-commerce industry are analyzed. Different simulation policies are designed based on government guidance, technological progress, logistics level, and market demand variables. The study finds that the development of E-commerce industry is affected by many factors and needs to be based on various factors. The government guidance, logistics level, and technological progress all have a greater positive effect on the development of the E-commerce industry. The government-guided coefficient is the most sensitive policy variable in industrial development, and the sensitivity of logistics level is higher than the level of technological progress.

\section{Acknowledgements}

This work was supported by the Modernization and Social Harmonious Development in Ethnic Regions: Research and Innovation Team of Northwest Minzu University (Grant No: 31920180101) and by the Key Laboratory of E-commerce of Ethnic information in Gansu Provincial (Grant No:MDS2017001) and Comprehensive experimental teaching center in Economics (Grant No. 2016XJSYJXSF2X-01-10019109).

\section{References}

[1] Liu Dwei, The status quo, problems and countermeasures of the development of E-commerce in China. Regional Economics, 2011(4)39-44

[2] Lu Xianghua, E-commerce ecosystem and its evolution path. Economic Management, 6(2009) 110-119.

[3] Guo Xuwen, E-commerce ecosystem structure, characteristics and evolution path, Business Times, 10 (2014) 71-76.

[4] Leisurely ginger, China's E-commerce industry SCP theory analysis, The contemporary economy 4 (2011) 116-120.

[5] Wang Fatao, Li Junqing, E-commerce industry chain model selection based on global value chain theory, China's circulation economy, 1 (2011) 45-51.

[6] Yu Changhong, The rise of E-commerce and its impact on the world economy, China Industrial Economy, 2 (2000)45-5.

[7] Liu Min, Chen Zheng, Research on E-commerce Development Measurement Index System, Statistics and Information Forum, 5 (2008) 20-28.

[8] Kannan, Yang Jian, Research on E-commerce development index system based on mutation progression evaluation method. Modern Intelligence, 2 (2014)3-8.

[9] Yu Dajin, Yang Shuling, The Dynamic Mechanism and Strategy Analysis of Agricultural E-commerce Development in China, Anhui Agricultural Sciences, 11 (2008) 4709-4711. 